\title{
A Possible Role for Posteriorly Directed Shear Loads in the Etiology of Degenerative Scoliosis \\ $+{ }^{1}$ Homminga, J; ${ }^{1}$ Lehr, A M; ${ }^{1}$ Meijer, G J M $;{ }^{2}$ Janssen, M M A; ${ }^{2}$ Schlosser, T P C; ${ }^{1}$ Verkerke, G J; ${ }^{2}$ Castelein, R \\ $+{ }^{1}$ Laboratory for Biomechanical Engineering, University of Twente, the Netherlands, ${ }^{2}$ University Medical Center Utrecht, the Netherlands
}

J.Homminga@utwente.nl

\section{INTRODUCTION:}

Scoliosis is a three-dimensional deformity of the human spine. Despite more than a century of dedicated research, its etiology remains largely unknown. The results of our past work, however, suggest a possible relation between the existing posteriorly directed shear loads on certain regions of the spine and the development of scoliosis. In modeling studies, we have shown that posteriorly directed shear loads do indeed occur in the human spine ${ }^{1}$. In in vitro studies, we found that these shear loads increase the transverse plane rotational instability of spinal segments ${ }^{2}$. And in in vivo studies we have shown that preexistent vertebral rotations are present and influenced by body position ${ }^{3,4}$. Based on these results, we believe that the upright spinal stance and the occurrence of posteriorly directed shear loads play a crucial role in the rotational instability of the human spine, and subsequently in scoliosis. Younger spines would then be most vulnerable as they are more rotationally unstable than adult spines ${ }^{5}$. While scoliosis is indeed most seen in adolescent spines, it also occurs in aged spines: degenerative scoliosis is a type of scoliosis that typically occurs in the thoraco-lumbar and lumbar spine of people over 65 years of age ${ }^{6}$. As people in this age group also typically have degeneration of their intervertebral discs, there appears to be a relation between disc degeneration and degenerative scoliosis. Disc degeneration has indeed been shown to cause increased rotational instability ${ }^{7,8}$, but the effect of additional shear loads on this behavior is yet unknown.

In this study we investigated whether the rotational instability of aged spines is related to disc degeneration.

\section{METHODS:}

Using our previously developed and validated L2-3 spinal motion segment model we studied the mechanical effects of disc degeneration on rotational instability ${ }^{5,9}$. This model contained two rigid vertebrae, an intervertebral discs (nucleus and fiber reinforced annulus), two facet joints, and all spinal ligaments (figure 1).

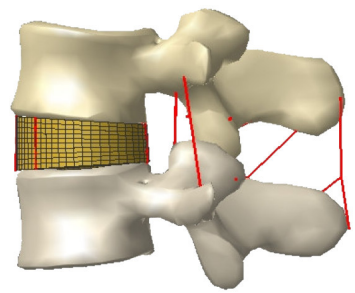

figure 1: FE-model of motion segment Disc degeneration was modeled by changing the properties of the intervertebral disc and ligaments in six sub-steps (table 1). As the etiology of disc degeneration is partly unclear and partly patient-specific, these sub-steps were not intended to represent subsequent steps in the disc degeneration process. Rather they were intended to represent possible cases of disc degeneration that may occur in certain patients. The modeled cases were: A. healthy disc, with hydrated nucleus and hydrated and fiber reinforced annulus. B. dehydrated nucleus (healthy annulus). C. dehydrated and fibrotic nucleus (healthy annulus). D. dehydrated and fibrotic nucleus and dehydrated annulus. E. dehydrated and fibrotic nucleus and dehydrated annulus including a reduction in disc height which in turn causes the fibers in the annulus and ligaments to become slack. F. dehydrated and chaotically fibrotic discus, including decreased disc height and slack ligaments (table 1).

\begin{tabular}{|c|c|c|c|c|c|c|}
\hline & $\begin{array}{l}\text { nucleus } \\
\mathrm{E}[\mathrm{MPa}]\end{array}$ & v & $\begin{array}{l}\text { annulus } \\
\mathrm{E}_{\text {base }}[\mathrm{MPa}]\end{array}$ & $v_{\text {base }}$ & $\mathrm{E}_{\text {fibers }}[\mathrm{MPa}]$ & disc height \\
\hline A & 1.0 & 0.49 & 2.0 & 0.45 & 450 & $100 \%$ \\
\hline $\mathrm{B}$ & 1.0 & 0.30 & 2.0 & 0.45 & 450 & $100 \%$ \\
\hline C & 8.0 & 0.30 & 2.0 & 0.45 & 450 & $100 \%$ \\
\hline D & 8.0 & 0.30 & 2.0 & 0.30 & 450 & $100 \%$ \\
\hline$E$ & 8.0 & 0.30 & 2.0 & 0.30 & 0 & $75 \%$ \\
\hline$F$ & 8.0 & 0.30 & 8.0 & 0.30 & 0 & $50 \%$ \\
\hline
\end{tabular}

The L3 vertebra was then fixed while the L2 vertebra was first loaded with either $150 \mathrm{~N}$ shear in posterior direction, no shear load, or $150 \mathrm{~N}$ shear in anterior direction. Subsequently the L2 vertebra was loaded with an additional $4 \mathrm{Nm}$ torsion moment in the transverse plane. The resulting torsion angles were used as a measure of the rotational instability of the segment under combined loading in shear and rotation; higher rotation angles indicate a higher instability.

\section{RESULTS:}

On average, for all disc degeneration cases, additional anterior shear forces decreased the rotation angle by $24 \%$, while additional posterior shear forces increased the rotation angle by $23 \%$ (figure 2 ).

Disc degeneration cases $\mathrm{B} / \mathrm{C} / \mathrm{D} / \mathrm{F}$ had only a small effect on the rotation angle of the motion segment under pure torsion moments (change $<9 \%$ ). Disc degeneration case E, however, showed a remarkable increase in rotation angle (84\%, figure 2 ).

Compared to a healthy disc, degeneration cases $\mathrm{B} / \mathrm{C} / \mathrm{D}$ only marginally affected the effect that shear forces have. Anterior shear forces decreased the rotation angle by $23-25 \%$, while posterior shear forces increased the rotation angle by $22-23 \%$. For disc degeneration case $\mathrm{E}$ the sensitivity to shear forces increased: anterior shear forces decreased the rotation angle by $29 \%$, while posterior shear forces increased the rotation angle by $31 \%$. For disc degeneration case $\mathrm{F}$ the sensitivity to shear forces decreased to levels below those for a healthy disc. Anterior shear forces decreased the rotation angle by $15 \%$, while posterior shear forces increased the rotation angle by $16 \%$ (figure 2 ).

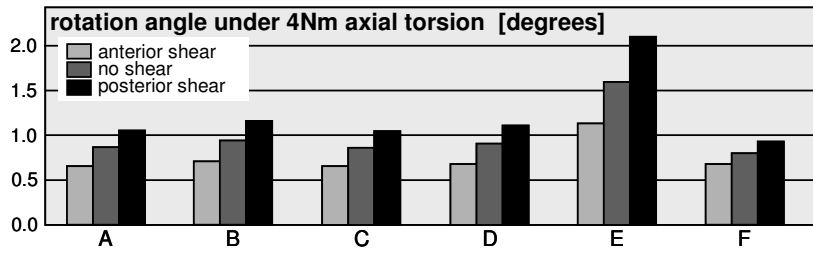

figure 2: torsional instability for different disc degeneration cases.

\section{DISCUSSION:}

Our numerical analyses show effects of anterior and posterior shear loads in combination with torsion moments that are very similar to those found in in vitro experiments ${ }^{2}$. Based on more detailed analyses of the loading of individual structures in the motion segment model, we found these effects to be mainly due to an earlier onset of facet joint contact under anterior shear than under posterior shear. The only other structure to behave significantly different under anterior and posterior shear are the facet joint capsules, these counteract the effect of the facet joint contact somewhat.

The disc degeneration cases where only the mechanical properties of the nucleus and annulus material are changed (cases $B / C / D$ ) change the behavior of the motion segment only slightly. This is somewhat in contrast to Tanaka et al. ${ }^{7}$ and Fujiwara et al. ${ }^{8}$, who found that mild stages of disc degeneration also affected the rotational instability. We believe this to be due to the fact that our "mild" degeneration cases (cases $\mathrm{B} / \mathrm{C} / \mathrm{D})$ do not include height loss, while their clinically mildly degenerated disc likely did include height loss.

In cases where decreased disc heights leads to slackness in the annulus fibers, the rotational instability is greatly increased as is the sensitivity to shear loads (case E). Further degeneration with decreased disc height as well as disorganization of the highly structured fiber structure in the annulus restores the rotational stability (case F). This restoration of rotational stability in later stages of disc degeneration was also observed by Tanaka et al. ${ }^{7}$ and Fujiwara et al. ${ }^{8}$

In conclusion we found that disc degeneration can indeed greatly increase the rotational instability, particularly under posterior shear loads. This increased rotational instability could very well be related to the onset and progression of degenerative scoliosis.

\section{SIGNIFICANCE:}

The treatment of scoliosis is hampered by the fact that its underlying causes remain largely unknown. This study is part of a series of studies aimed at unraveling the causes of scoliosis.

\section{REFERENCES}

1. Castelein et al. Med.Hypotheses. 65:501-508, 2005. 2. Kouwenhoven et al. Spine 32: 25452550, 2007. 3. Kouwenhoven et al. Spine 31:1467-1472, 2006. 4. Janssen et al. Eur.Spine J. 19:1728-1734, 2010. 5. Meijer et al. J.Biomech. 43:1590-1597, 2010. 6. Aebi. Eur.Spine J. 14:925-948, 2005. 7. Tanaka, Spine J. 1:47-56, 2001. 8. Fujiwara, Spine. 25:3036, 2000 9. Meijer et al. Spine 36:929-935, 2011 